\title{
ALMOST ALTERNATING DIAGRAMS AND FIBERED LINKS IN $S^{3}$
}

\author{
HIROSHI GODA, MIKAMI HIRASAWA AND RYOSUKE YAMAMOTO
}

\section{IntRODUCTION AND STATEMENTS OF RESULTS}

The concept of Murasugi sum (for the definition, see Section 2) of Seifert surfaces in the 3 -sphere $S^{3}$ was introduced by K. Murasugi, and it has been playing important roles in the studies of Seifert surfaces and links. The Murasugi sum is known to be natural in many senses, and in particular the following is known. (We say that a Seifert surface $R$ is a fiber surface if $\partial R$ is a fibered link and $R$ realizes the fiber.)

Theorem 1.1 (四, Theorem 3.1]). Let $R$ be a Murasugi sum of $R_{1}$ and $R_{2}$. Then $R$ is a fiber surface if and only if both $R_{1}$ and $R_{2}$ are fiber surfaces.

On the other hand, the concept of alternating link has also been important in knot theory. It has been known that there are some relationships between alternating diagrams and the Seifert surfaces obtained by applying Seifert's algorithm to them. For example, if a link diagram $D$ is alternating, then the Seifert surface obtained from $D$ by the algorithm is of minimal genus, [3, 7].

In [4], D. Gabai gave a geometric proof to the following theorem, which also follows from [9] and [11]. Note that if $L$ is fibered, then minimal genus Seifert surfaces for $L$ are unique up to isotopy and the fiber is realized by the minimal genus surface.

Theorem 1.2 (画, Theorem 5.1]). Let L be an oriented link with an alternating diagram $D$. $L$ is a fibered link if and only if the surface $R$ obtained by applying Seifert's algorithm to $D$ is connected and (obviously) desums into a union of Hopf bands.

We say that a Seifert surface $R\left(\subset S^{3}\right)$ desums into $R_{1}, \ldots, R_{n}$ if $R$ is a Murasugi sum of them. Especially, if $R$ is obtained by successively plumbing (i.e., 4-Murasugi summing) finite number of Hopf bands to a disk, we call $R$ a Hopf plumbing.

Actually, the 'only if' part of Theorem 1.2 can be strengthened as in the following theorem, which follows from Propositions 5.1 and 5.2 .

The first author was supported in part by Hyogo Science and Technology Association. The second author is supported by Fellowships of the Japan Society for the Promotion of Science for Japanese Junior Scientists. 
Theorem 1.3. Let $L$ be an oriented link with an alternating diagram $D . L$ is a fibered link if and only if the surface $R$ obtained by applying Seifert's algorithm to $D$ is a Hopf plumbing. Moreover, $R$ is a fiber surface if and only if $R$ is deformed into a disk by successively cutting one of a pair of 'parallel bands' (defined in Section 5).

In [1], C. Adams et al. generalized the concept of alternating links and introduced the concept of almost alternating links. A diagram $D$ in $S^{2}$ is called almost alternating (resp. 2-almost alternating) if $D$ becomes an alternating diagram after one crossing change (resp. two crossing changes). A link $L$ in $S^{3}$ is called almost alternating if $L$ is not alternating but admits an almost alternating diagram. If $D$ is an almost alternating diagram, the specific crossing to change is called the dealternator and we call the other crossings the alternators.

In this paper, we extend Theorems 1.2 and 1.3 to almost alternating links. Note that almost alternating diagrams, however, do not always yield a minimal genus Seifert surface via Seifert's algorithm. Our first result is as follows:

Theorem 1.4. Let $D$ be an almost alternating diagram, and $R$ a Seifert surface obtained by applying Seifert's algorithm to $D$. Then, $R$ is a fiber surface if and only if $R$ is connected and desums into a union of Hopf bands.

In section 5, we show a stronger version of Theorem 1.4 as below, by using Corollary 1.6 obtained from the arguments in the proof of Theorem 1.4 .

Theorem 1.5. Let $R$ be a Seifert surface obtained by applying Seifert's algorithm to an almost alternating diagram. Then, $R$ is a fiber surface if and only if $R$ is a Hopf plumbing.

As a corollary of the proof of Theorem 1.4, we obtain a practical algorithm to determine whether or not a given almost alternating diagram yields a fiber surface via Seifert's algorithm. We use this to prove Theorem 1.5. We say that a diagram $D$ is unnested if $D$ has no Seifert circle which contains another circle in both of its complementary region. Otherwise we say $D$ is nested.

Corollary 1.6. Let $D$ be an almost alternating diagram and $R$ a Seifert surface obtained from $D$ by Seifert's algorithm. Then $R$ is a fiber surface if and only if $R$ is connected and desums into a union of Hopf bands by repeating of the following decompositions;

(1) a Murasugi decomposition along a nested Seifert circle,

(2) a prime decomposition, and 
(3) Murasugi decompositions of type (A) and (B) in Figure 1.1, where each decomposition yields Seifert surfaces with first Betti numbers smaller than that of $R$.

\section{Figure 1.1}

Proof. In the proof of Theorems 1.2 (see [甘, p.533]) and 1.4, we explicitly show how we can desum such $R$ into surfaces of smaller first Betti numbers. All necessary decompositions are covered in the above three.

1.6

In [5], J. Harer proved that every fiber surface in $S^{3}$ results from a disk by a sequence of elementary changes as follows:

(a) plumb on a Hopf band,

(b) deplumb a Hopf band, and

(c) perform a Dehn twist about a suitable unknotted curve in the fiber.

Then he asked whether changes of either type (b) or (c) can be omitted, and any fiber surface can be realized only using changes of the remaining two types.

So it is worthy presenting the following partial affirmative answer as a corollary, which immediately follows from Theorem 1.5 and Propositions 5.1 and 5.2.

Corollary 1.7. Let $R_{1}$ and $R_{2}$ be any fiber surface obtained by applying Seifert's algorithm to an alternating or almost alternating diagram. Then $R_{1}$ and $R_{2}$ can be changed into each other by plumbing and deplumbing Hopf bands.

We say that a Hopf hand $B$ is positive (resp. negative) if the linking number of $\partial B$ is 1 (resp. -1). By the following fact together with an observation of the way fiber surfaces deplumb in the proof of Theorem 1.4 (see Section 4) and Theorem 1.5 (see Section 5), we have the following Corollary:

Corollary 1.8. Let $D$ be an unnested almost alternating diagram such that the sign of the dealternator is negative. Suppose the surface $R$ obtained from $D$ by Seifert's algorithm is a fiber surface. Then $R$ is a plumbing of positive Hopf bands.

Fact 1.9. Suppose a diagram $D$ is unnested. Then $D$ is alternating (resp. almost alternating) if and only if all the crossings of D have the same sign (resp. the same sign except exactly one crossing).

This paper is organized as follows; Section 2 is for preliminaries. In Section 3, we give an example for our theorem. We also show that our theorem can not be extended to 2-almost alternating diagrams, i.e., (1) we recall Gabai's example (in [4]) of a 2-almost 
alternating diagram for a link whose Seifert surface obtained by Seifert's algorithm is a fiber surface that is not a nontrivial Murasugi sum, and (2) we give examples of 2-almost alternating diagrams for knots whose Seifert surfaces obtained by Seifert's algorithm are fiber surfaces that are not Hopf plumbing. In Sections 4 and 5, we prove Theorems 1.4 and 1.5 respectively.

\section{Preliminaries}

For the definitions of standard terms of sutured manifolds, see [曰, p.520]. We say that a sutured manifold $(M, \gamma)$ is a product sutured manifold if $(M, \gamma)$ is homeomorphic to $(R \times I, \partial R \times I)$ with $R_{+}(\gamma)=R \times\{1\}, R_{-}(\gamma)=R \times\{0\}$, where $R$ is a compact oriented surface with no closed components and $I$ is the unit interval $[0,1]$.

The exterior $E(L)$ of a link $L$ in $S^{3}$ is the closure of $S^{3}-N\left(L ; S^{3}\right)$. If $R$ is a Seifert surface for $L$, we may assume $R \cap E(L)$ is homeomorphic to $R$, and often abbreviate $R \cap E(L)$ as $R$.

Let $R$ be a Seifert surface for $L$ in $S^{3}$. The product sutured manifold $(M, \gamma)=$ $(R \times I, \partial R \times I)$ is called the sutured manifold obtained from $R$ and the sutured manifold $(N, \delta)=(E(L)-\operatorname{Int} M, \partial E(L)-\operatorname{Int} \gamma)$ is the complementary sutured manifold for $R$ (or for $(M, \gamma))$.

Note that $R$ is a fiber surface if and only if the complementary sutured manifold for $R$ is a product sutured manifold.

A product decomposition [4] is a sutured manifold decomposition

$$
\left(M_{1}, \gamma_{1}\right) \stackrel{B}{\longrightarrow}\left(M_{2}, \gamma_{2}\right)
$$

where $B$ is a disk properly embedded in $M_{1}$ such that $B \cap s\left(\gamma_{1}\right)=(2$ points $), M_{2}=$ $M_{1}-\operatorname{Int} N(B)$ and that $s\left(\gamma_{2}\right)$ is obtained by extending $s\left(\gamma_{1}\right)-\operatorname{Int} N(B)$ in the natural way (Figure 2.1 (a)). The disk $B$ is called a product disk.

Dually, $C$-product decomposition is the operation

$$
\left(M_{1}, \gamma_{1}\right) \stackrel{E}{\longrightarrow}\left(M_{2}, \gamma_{2}\right)
$$

where $E$ is a disk properly embedded in $S^{3}-\operatorname{Int} M_{1}$ such that $E \cap s\left(\gamma_{1}\right)=(2$ points), $M_{2}$ is obtained from $M_{1}$ by attaching the 2-handle $N(E)$ and that $s\left(\gamma_{2}\right)$ is obtained by extending $s\left(\gamma_{1}\right)-\operatorname{Int} N(E)$ in the natural way (Figure $2.1(\mathrm{~b})$ ). The disk $E$ is called a C-product disk.

Figure 2.1 
Definition. Let $R$ be a Seifert surface for a link $L$. We say that $R$ has a product decomposition if there exists a sequence of $C$-product decompositions

$$
(R \times I, \partial R \times I)=\left(M_{0}, \gamma_{0}\right) \stackrel{E_{1}}{\longrightarrow}\left(M_{1}, \gamma_{1}\right) \stackrel{E_{2}}{\longrightarrow} \cdots \stackrel{E_{p}}{\longrightarrow}\left(M_{p}, \gamma_{p}\right),
$$

where the complementary sutured manifold for $\left(M_{p}, \gamma_{p}\right)$ is a union of 3-balls each with a single suture.

As a criterion to detect a fiber surface, Gabai has shown the following:

Theorem 2.1 (四, Theorem 1.9]). Let $L$ be an oriented link in $S^{3}$, and $R$ a Seifert surface for $L$. Then, $L$ is a fibered link with fiber $R$ if and only if $R$ has a product decomposition.

We note that in Section 4, the existence of a $C$-product decomposition $\left(M_{0}, \gamma_{0}\right) \stackrel{E_{1}}{\longrightarrow}$ $\left(M_{1}, \gamma_{1}\right)$ together with the $C$-product disk $E_{1}$ is important.

Definition. A surface $R\left(\subset S^{3}\right)$ is a $2 n$-Murasugi sum of two surfaces $R_{1}$ and $R_{2}$ in $S^{3}$ if the following conditions are satisfied;

1. $R=R_{1} \cup_{\Delta} R_{2}$, where $\Delta$ is a $2 n$-gon, i.e., $\partial \Delta=\mu_{1} \cup \nu_{1} \cup \ldots \cup \mu_{n} \cup \nu_{n}$ (possibly $n=1)$, where $\mu_{i}\left(\right.$ resp. $\left.\nu_{i}\right)$ is an arc properly embedded in $R_{1}\left(\right.$ resp. $\left.R_{2}\right)$.

2. There exist 3-balls $B_{1}$ and $B_{2}$ in $S^{3}$ such that:

(i) $B_{1} \cup B_{2}=S^{3}, B_{1} \cap B_{2}=\partial B_{1}=\partial B_{2}=S^{2}$ : a 2-sphere,

(ii) $R_{1} \subset B_{1}, R_{2} \subset B_{2}$ and $R_{1} \cap S^{2}=R_{2} \cap S^{2}=\Delta$.

The 2-Murasugi sum is known as the connected sum, and the 4-Murasugi sum is known as the plumbing.

$$
\text { Figure 2.2 }
$$

Concerning alternating and almost alternating tangles, we can confirm the following facts.

Fact 2.2. Suppose a link diagram $D$ is a tangle sum of two tangle diagrams $D_{1}$ and $D_{2}$. If $D$ is alternating, then both $D_{1}$ and $D_{2}$ are alternating. And if $D$ is almost alternating, then one of them, say, $D_{1}$ is alternating and $D_{2}$ is almost alternating.

Fact 2.3. By connecting neighboring strands running out of an alternating (resp. almost alternating) tangle diagram, we obtain an alternating (resp. almost alternating) link diagram. See Figure 2.3.

Figure 2.3 
Then by these two facts, we can confirm the following propositions. Let $R$ be a Seifert surface obtained by applying Seifert's algorithm to a diagram $D$.

Proposition 2.4. If an almost alternating diagram $D$ is a connected sum of two diagrams, then one of them, say, $D_{1}$ is alternating and the other, say, $D_{2}$ is almost alternating. The Seifert surface $R$ is a 2-Murasugi sum of $R_{1}$ and $R_{2}$, where $R_{i}$ is obtained from $D_{i}$.

Proposition 2.5. Suppose that an almost alternating diagram $D$ has a nested Seifert circle $C$. Then, along the disk bounded by $C, R$ is a Murasugi sum of $R_{1}$ and $R_{2}$, where $R_{1}$ (resp. $R_{2}$ ) is obtained from an alternating (resp. almost alternating) diagram.

Proposition 2.6. Suppose that $R$ desums into two surfaces $R_{1}$ and $R_{2}$ as illustrated in Figure 1.1, where the left figures in $(A)$ and $(B)$ are both almost alternating. Then $R_{i}$ $(i=1,2)$ is obtained from an alternating or almost alternating diagram.

\section{EXAMPLES}

In this section, we present some examples. Example 3.1 is for Theorems 1.4 and 1.5 . Examples 3.2 and 3.3 show our Theorems 1.4 and 1.5 can not be extended to 2-almost alternating diagrams. For the names of knots, refer to Rolfsen's book [10].

Example 3.1. Figure 3.1 depicts an almost alternating diagram for the knot $10_{151}$, together with a fiber surface $R$ obtained by Seifert's algorithm. We can observe that $R$ desums into a union of Hopf bands and is a Hopf plumbing.

$$
\text { Figure 3.1 }
$$

Example 3.2. Let $R$ be the Seifert surface obtained by applying Seifert's algorithm to the oriented pretzel link diagram of type $(2,-2,2 p)$ as in Figure 3.2, where $p \neq 0 . R$ is a fiber surface but does not desum into a union of Hopf bands.

We note that this example has been known in [4] as a fiber surface for a link which does not admit a non-trivial Murasugi sum.

$$
\text { Figure } 3.2
$$

Example 3.3. Figure 3.3 depicts 2 -almost alternating diagrams for the knots $9_{42}, 9_{44}$ and $9_{45}$. By applying Seifert's algorithm to them, we obtain fiber surfaces, which are not Hopf plumbings. This can be shown by the following proposition and direct calculations of genera and the Conway polynomials of these knots. 
Proposition 3.4 ([6, Theorem 3]). If a fibered knot $K$ of genus 2 can be constructed by plumbing Hopf bands, then the Conway polynomial $\nabla_{K}(z)$ of $K$ satisfies the following;

$$
\nabla_{K}(z) \neq \begin{cases}1+c_{1} z^{2}+z^{4} & \text { for } c_{1}=0 \bmod 4 \\ 1+c_{1} z^{2}-z^{4} & \text { for } c_{1}=2 \bmod 4\end{cases}
$$

\section{Figure 3.3}

\section{Proof of Theorem 1.4}

Since the 'if' part is shown by Theorem 1.1, we show the 'only if' part. Let $D$ be an almost alternating diagram for a link $L\left(\subset S^{3}\right)$ on the level 2-sphere $S^{2}$ and let $R$ be a Seifert surface obtained by applying Seifert's algorithm to $D$.

Note that if a diagram $D$ is unnested, then Seifert's algorithm uniquely yields a Seifert surface. We say that a Seifert surface $R$ is flat if $R$ is obtained from an unnested diagram and thus lies in $S^{2}$ except in the neighborhood of each crossing.

Suppose that $R$ is a fiber surface. Since any fiber surface is connected, we can assume $D$ is connected.

Suppose $D$ is nested. Then, by Theorem 1.1, $R$ desums into fiber surfaces $R_{1}$ and $R_{2}$. Moreover, by Proposition 2.5, one of them, say, $R_{1}$ is obtained from an alternating diagram and $R_{2}$ from an almost alternating diagram. By Theorem 1.2, $R_{1}$ desums into a union of Hopf bands. Therefore, we may assume that $D$ is unnested.

Similarly, by Proposition 2.4, we may assume that $D$ is prime, and in particular, reduced.

Now we prove the theorem by induction on the first Betti number $\beta_{1}$ of $R$, where $R$ is a fiber surface obtained by applying Seifert's algorithm to a connected unnested prime almost alternating diagram $D$. If $\beta_{1}=1$, then $R$ is an unknotted annulus and $D$ has $n$ crossings which are of the same sign except exactly one crossing. Note that $R$ is a fiber surface if and only if $n=4$, in which case $R$ is a Hopf band. Hence we have the conclusion.

Then we assume that the theorem holds when $\beta_{1}(R)<k$ and prove the theorem for $R$ with $1 \leq \beta_{1}(R)=k$.

The main method of the proof is to examine the $C$-product disk for the sutured manifold obtained from $R$ and grasp a local picture where we can desum $R$ into surfaces $R_{1}$ and $R_{2}$ obtained by the algorithm with smaller first Betti numbers. In each case, it is easy to confirm that $D_{i}(i=1,2)$ is an alternating or almost alternating diagram, 
that is, they satisfy the assumption of the induction (see Corollary 1.6 and Proposition 2.6).

Let $(M, \gamma)$ be the sutured manifold obtained from $R$. We identify $s(\gamma)$ as $L$. Let $E$ be a $C$-product disk for $(M, \gamma)$, i.e., $E$ is properly embedded in $S^{3}-\operatorname{Int} M$ so that $E \cap L=$ (2 points). We may suppose that $E$ is non-boundary-parallel, and assume that $\left|E \cap S^{2}\right|$ is minimal among all such disks. Further, we may assume by isotopy that $\partial E \cap L$ occurs only in small neighborhoods of the crossings of $D$. Similarly, we can assume that $\partial E \cap S^{2}$ occurs only in small neighborhoods of the crossings. For convenience, we say that $\partial E \cap L$ and $\partial E \cap S^{2}$ occur at the crossings.

Case A. $E \cap S^{2}=\emptyset$.

If $\partial E \cap L$ occurs at one crossing, then $E$ is boundary parallel, a contradiction. Thus, we suppose that $\partial E \cap L$ occurs at two crossings (see Figure 4.1). If both crossings are alternators, we see that $R$ is a plumbing of flat surfaces, one of which is obtained from an unnested almost alternating diagram and has first Betti number smaller than $k$. If one crossing is the dealternator, we also see that $R$ is a plumbing of surfaces, one of which is compressible and hence not a fiber surface, a contradiction to Theorem 1.1 .

\section{Figure 4.1}

Case B. $E \cap S^{2} \neq \emptyset$.

Label the crossings with $\mathfrak{a}_{0}, \mathfrak{a}_{1}, \ldots, \mathfrak{a}_{w-1}$ so that the dealternator has $\mathfrak{a}_{0}$. By standard innermost circle argument, we may assume, by the minimality of $\left|E \cap S^{2}\right|$, that $E \cap S^{2}$ consists of arcs. Let $\alpha$ be an arc of $E \cap S^{2}$. By assumption, each endpoint of $\alpha$ lies in a neighborhood of a crossing and hence is accordingly labeled. Then the label of $\alpha$ is a pair $\left(\mathfrak{a}_{i}, \mathfrak{a}_{j}\right)$ of the labels of $\partial \alpha$. The two points of $\partial E \cap L$ are also labeled according to the crossings at which $\partial E \cap L$ occurs.

Lemma 4.1. For any arc $\alpha$ of $E \cap S^{2}$ with label $\left(\mathfrak{a}_{i}, \mathfrak{a}_{j}\right)$, we have $i \neq j$.

Proof. If both of the endpoints of $\alpha$ occur at the same crossing $\mathfrak{a}_{i}$, we can observe that one of the two cases in Figure 4.2 occurs. In Figure 4.2 (a), $D$ is non-prime. In Figure 4.2 (b), there exists an arc $\alpha^{\prime}$ of $E \cap S^{2}$ in $S^{2}-\operatorname{Int} M$ such that the endpoints of $\alpha^{\prime}$ occur at the same crossing $\mathfrak{a}_{i}$, and that $\alpha^{\prime}$ cuts off a disk $H$ from $S^{2}-\operatorname{Int} M$ with Int $H \cap\left(E \cap S^{2}\right)=\emptyset$. We can surgery $E$ along $H$ so that we obtain two disks $E_{1}, E_{2}$ properly embedded in $S^{3}-\operatorname{Int} M$. Since both endpoints of $\alpha^{\prime}$ are in $R_{+}(\gamma)$ (or $R_{-}(\gamma)$ ), one of them, say $E_{1}$, intersects $L$ twice. Since $E$ is non-boundary-parallel, so is $E_{1}$ or $E_{2}$. If $E_{2}$ is, then it yields a compressing disk for $R$, a contradiction. Hence $E_{1}$ is a non-boundary-parallel $C$-product disk with $\left|E_{1} \cap S^{2}\right|<\left|E \cap S^{2}\right|$, a contradiction. 4.1 
Figure 4.2

We look at an outermost disk $F \subset E$ (i.e., $F$ is the closure of a component of $E-S^{2}$ such that $F \cap S^{2}$ is connected).

Lemma 4.2. Let $\alpha$ be an outermost arc of $E \cap S^{2}$ with label $\left(\mathfrak{a}_{i}, \mathfrak{a}_{j}\right)$, cutting an outermost disk $F$ off $E$. Then we may assume that $i \neq j$ and that $i$ or $j=0$ if $F \cap L=\emptyset$ or $(a$ point).

Proof. By Lemma 4.1, we have $i \neq j$. Suppose $i \neq 0$ and $j \neq 0$. If $|F \cap L|=0, R$ is non-prime, a contradiction (Figure 4.3 (a)). If $|F \cap L|=1$, then either $\left|E \cap S^{2}\right|$ is not minimal, or $R$ is a plumbing (Figures 4.3 (b) and (c)).

4.2

$$
\text { Figure } 4.3
$$

Concerning outermost disks, we have two cases.

Case B-1. There exists an outermost disk $F$ with $F \cap L=$ (a point). Let $\alpha$ be the arc $F \cap S^{2}(\subset E)$. By Lemma 4.2, we assume the label of $\alpha$ is $\left(\mathfrak{a}_{0}, \mathfrak{a}_{j}\right)$, where $j \neq 0$. Let $\mathfrak{a}_{k}$ be the label of the point of $\partial E \cap L$ on $F$. Then we have three cases; Subcase 1: $k=0$, Subcase 2: $k=j$, and Subcase 3: $k \neq 0$ and $k \neq j$. In Subcases 1 and 2, D is non-prime (Figure $4.4(\mathrm{a})$ ). In Subcase 3, $R$ is a plumbing or we can isotope $E$ so that the outermost disk of Case B-1 is replaced by an outermost disk of Case B-2 (Figure $4.4(\mathrm{~b})$ ).

$$
\text { Figure } 4.4
$$

Lemma 4.3. We may assume there exists no outermost disk of Case B-1.

Proof. If the latter situation of Subcase 3 above occurs, we can view the above isotopy of $E$ as sliding a point of $\partial E \cap L$ out of $F$. Hence by repeating the above isotopies at most twice, we may eliminate outermost disks of Case B-1. 4.3

Case B-2. There exists an outermost disk $F$ with $F \cap L=\emptyset$.

By Lemma 4.2, we may assume $\alpha=F \cap S^{2}$ appears as in Figure 4.5. We note that outermost disks of this kind are typically found in the complementary sutured manifold for the fiber surface in Figure 3.2, which is obtained from a 2-almost alternating diagram. The rest of the proof really depends on the almost-alternatingness of $D$.

$$
\text { Figure 4.5 }
$$


Lemma 4.4. For any arc $\beta$ of $\partial E-\left(E \cap S^{2}\right)$, if $\beta \cap L=\emptyset$, then the endpoints of $\beta$ have different labels.

Proof. Suppose the two endpoints of $\beta$ have the same label. Then $\beta$ appears as in Figure 4.6 and we can isotope $E$ to a $C$-product disk $E^{\prime}$ such that $\left|E^{\prime} \cap S^{2}\right|=\left|E \cap S^{2}\right|-1$, a contradiction.

\section{Figure 4.6}

Lemma 4.5. Suppose E locally appears as in Figure $4.7(a)$, i.e., $\mathfrak{a}_{i}, \mathfrak{a}_{j}$ and $\mathfrak{a}_{k}$ are the labels of points of $\left(\partial E \cap S^{2}\right) \cup(\partial E \cap L)$ sequential in $\partial E$ such that the former two points are connected by an outermost arc of $E \cap S^{2}$ and the last is a point of $\partial E \cap S^{2}$. Then $i, j, k$ are mutually different.

Proof. By Lemma 4.2, we have $i \neq j$ and $i$ or $j=0$. Suppose $i=k$. Then we can find a compressing disk for $R$ in Figure 4.7 (b), a contradiction. By Lemma 4.4, we have $j \neq k$.

\section{Figure 4.7}

Lemma 4.6. We may assume that the following situation never occurs; The disk E locally appears as in Figure 4.8 (a), i.e., $\mathfrak{a}_{i}, \mathfrak{a}_{j}, \mathfrak{a}_{k}$ and $\mathfrak{a}_{l}$ are the labels of points of $\left(\partial E \cap S^{2}\right) \cup(\partial E \cap L)$ sequential in $\partial E$ such that the former two points and the latter two are respectively connected by outermost arcs $\alpha_{1}$ and $\alpha_{2}$ of $E \cap S^{2}$.

Proof. By Lemmas 4.2, 4.4 and 4.5, we may assume that $i=l=0$ and $j \neq k$. Then we obtain the conclusion, since in Figure 4.8 (b) $\alpha_{2}$ can not coexist with the arc of $\partial E-S^{2}$ connecting $\mathfrak{a}_{k}$ and $\mathfrak{a}_{l}$.

4.6

\section{Figure 4.8}

Lemma 4.7. Suppose E locally appears as in Figure 4.9 (a), i.e., $\mathfrak{a}_{i}, \mathfrak{a}_{j}$ and $\mathfrak{a}_{k}$ are the labels of points of $\left(\partial E \cap S^{2}\right) \cup(\partial E \cap L)$ sequential in $\partial E$ such that the former two points are connected by an outermost arc of $E \cap S^{2}$ and the third point is of $\partial E \cap L$. Then we may assume $i, j, k$ are mutually different. 
Proof. By Lemma 4.2, we have $i \neq j$, and $i$ or $j=0$. If $k=i$, then $R$ is compressible, a contradiction (see Figure $4.9(\mathrm{~b})$ ). If $k=j$, we can reduce $\left|E \cap S^{2}\right|$ by isotopy, a contradiction (see Figure 4.9 (c)).

4.7

$$
\text { Figure 4.9 }
$$

Lemma 4.8. Let $\mathfrak{a}_{l}$ be the label of the point $x$ of $\partial E \cap L$. Suppose that the two points adjacent to $x$ in $\partial E$ are points of $\partial E \cap S^{2}$. Then the two adjacent points do not have the same label except for the case where they are both $\mathfrak{a}_{l}$.

Proof. Suppose the two points have the same label $\mathfrak{a}_{i}\left(\neq \mathfrak{a}_{l}\right)$. By Lemma 4.1, we may assume that they are not connected by an arc of $E \cap S^{2}$. Then we can find a $C$-product disk $E^{\prime}$ in Figure 4.10 such that $\left|E^{\prime} \cap S^{2}\right|=0$, a contradiction.

\section{Figure 4.10}

Similarly we have the following lemma.

Lemma 4.9. Suppose E locally appears as in Figure 4.11(a), i.e., $\mathfrak{a}_{i}, \mathfrak{a}_{j}, \mathfrak{a}_{k}$ and $\mathfrak{a}_{l}$ are the labels of points of $\left(\partial E \cap S^{2}\right) \cup(\partial E \cap L)$ sequential in $\partial E$ such that the former two points are connected by an outermost arc of $E \cap S^{2}$, the third point is of $\partial E \cap L$ and that the fourth is of $\partial E \cap S^{2}$. If $k \neq l$, then $i, j, k$ and $l$ are mutually different.

Proof. By Lemma 4.7, we may assume $i, j, k$ are mutually different. Then by Lemma 4.8 , we have $l \neq j$. Suppose $l \neq k$ and $l=i$. Then by Lemma $4.2, \mathfrak{a}_{i}=\mathfrak{a}_{l}=\mathfrak{a}_{0}$ (Figure 4.11 (b)) or $\mathfrak{a}_{j}=\mathfrak{a}_{0}$ (c). In either case, we can find a $C$-product disk $E^{\prime}$ such that $\left|E^{\prime} \cap S^{2}\right|=0$, a contradiction.

$$
\text { Figure } 4.11
$$

Lemma 4.10. We may assume the following situation never occurs; The disk E locally appears as in Figure 4.12 (a), i.e., $\mathfrak{a}_{i}, \mathfrak{a}_{j}, \mathfrak{a}_{k}, \mathfrak{a}_{l}$ and $\mathfrak{a}_{m}$ are the labels of points of ( $\partial E \cap$ $\left.S^{2}\right) \cup(\partial E \cap L)$ sequential in $\partial E$ such that the first two points and the last two points are respectively connected by an outermost arc of $E \cap S^{2}$, and that the third point is of $\partial E \cap L$.

Proof. By Lemma 4.7, $k, l$ and $m$ are mutually different and hence by Lemma $4.9, i, j, k$ and $l$ are mutually different. By Lemma 4.2, $i$ or $j=0$ and $l$ or $m=0$, and hence $m=0$, and by symmetry, we have $i=0$. Then $\mathfrak{a}_{i}, \mathfrak{a}_{j}, \mathfrak{a}_{k}, \mathfrak{a}_{l}$ and $\mathfrak{a}_{m}$ appear as in Figure 
4.12 (b), where we can find a $C$-product disk $E^{\prime}$ such that $\left|E \cap S^{2}\right|>\left|E^{\prime} \cap S^{2}\right|=1$, a contradiction. We note that $E^{\prime} \cap L$ occurs at $\mathfrak{a}_{k}$ and $\mathfrak{a}_{l}$.

4.10

$$
\text { Figure } 4.12
$$

An $\operatorname{arc} \varepsilon$ of $E \cap S^{2}$ is said to be of level 2 if it is not outermost and, for one component $E_{1}$ of $E-\varepsilon, E_{1} \cap S^{2}$ is a union of outermost arcs in $E \cap S^{2}$. Suppose there is no arc of level 2. Then by Lemmas 4.3, 4.6 and 4.10, we see that $E \cap S^{2}$ consists of only one arc $\alpha$ such that one component of $E-\alpha$ contains the two points of $\partial E \cap L$. Let $\left(\mathfrak{a}_{0}, \mathfrak{a}_{j}\right)$ be the label of $\alpha$, and let $\mathfrak{a}_{k}$ and $\mathfrak{a}_{l}$ be the labels of the two points of $\partial E \cap L$, where $\mathfrak{a}_{0}, \mathfrak{a}_{j}, \mathfrak{a}_{k}$ and $\mathfrak{a}_{l}$ appear in this order in $\partial E$. If $l=k$, then we can isotope $E$ so that $E \cap L=\emptyset$ and we have a compressing disk for $R$, for $E$ is not boundary parallel, a contradiction. Hence by Lemma 4.7, we can assume $j, k, l, 0$ are mutually different. In this case, $R$ desums into three surfaces $R_{1}, R_{2}$ and $R_{3}$ obtained by applying Seifert's algorithm to the almost alternating diagrams $D_{1}, D_{2}$ and $D_{3}$ respectively (Figure 4.13).

$$
\text { Figure } 4.13
$$

Hence we assume there is an arc of level 2. Then by Lemmas 4.3, 4.6 and 4.10, we see that there exists an $\operatorname{arc} \varepsilon$ of level 2 such that one disk $E_{1}$ cut by $\varepsilon$ off $E$ contains one outermost arc of $E \cap S^{2}$ and satisfies one of the following conditions;

$\left({ }^{*}\right) E_{1} \cap L=\emptyset$, (**) $E_{1} \cap L=$ a point.

If $E_{1}$ satisfies $\left(^{*}\right)$, by Lemmas 4.1 and 4.5 , all four labels of points of $E_{1} \cap S^{2}$ are mutually different. Then, we can see that $D$ is non-prime or $R$ is a plumbing (Figure 4.14).

$$
\text { Figure 4.14 }
$$

Thus we have:

Lemma 4.11. We may assume that there is no arc of level 2 which cuts a disk $E_{1}$ off $E$ such that $E_{1}$ contains only one (outermost) arc of $E \cap S^{2}$ and that $E_{1} \cap L=\emptyset$.

In what follows, we assume that there exists an arc $\varepsilon$ of level 2 which cuts off $E$ a disk $E_{1}$ containing one outermost arc of $E \cap S^{2}$ and satisfying (**).

By Lemma 4.3, we may suppose that $E_{1}$ appears as in Figure 4.15 (a) with labels $\mathfrak{a}_{i}, \mathfrak{a}_{j}, \mathfrak{a}_{k}, \mathfrak{a}_{l}$ and $\mathfrak{a}_{m}$.

Lemma 4.12. All five labels in $E_{1}$ are mutually different. 
Proof. By Lemma 4.5, $i, j, k$ are mutually different. By Lemma 4.7, $l \neq k$ and $l \neq j$. We see $l \neq i$, for if not, $R$ appears as in Figure 4.15 (b) or (c), and in either case, $R$ is compressible, a contradiction. Now we have seen that $i, j, k, l$ are mutually different. Next suppose $m=l$. Then $R$ appears as in Figure 4.15 (d) or (e). In Figure 4.15 (d), $R$ is a plumbing or we can isotope $E$ to reduce $\left|E \cap S^{2}\right|$. In Figure 4.15 (e), $R$ is a Murasugi sum or we can isotope $R$ so that $D$ becomes an alternating diagram and the result follows from Theorem 1.2. Hence we can assume $m \neq l$ and by Lemma 4.9, we see that $j, k, l, m$ are mutually different and by Lemma $4.1, m \neq i$.

4.12

\section{Figure 4.15}

Lemma 4.13. We may assume $\mathfrak{a}_{j}=\mathfrak{a}_{0}$.

Proof. If not, $\mathfrak{a}_{k}=\mathfrak{a}_{0}$ by Lemma 4.2. Then $R$ is a 6-Murasugi sum as in Figure 4.16. 4.13

$$
\text { Figure 4.16 }
$$

Lemma 4.14. Let $\varepsilon$ and $E_{1}$ be as above. Then there is no arc $\varepsilon^{\prime}$ of $E \cap S^{2}$ as in Figure 4.17 which cuts a disk $E_{2}$ off $E$ with the following conditions:

1. $E_{1} \subset E_{2}$,

2. $\left(\operatorname{Int} E_{2}-E_{1}\right) \cap\left(E \cap S^{2}\right)=\emptyset$,

3. $E_{2} \cap L=E_{1} \cap L=(1$ point $)$.

Figure 4.17

Proof. By Lemmas 4.12 and 4.13, we may assume that $E_{1}$ appears as in Figure 4.18. Recall that $R$ is flat. Suppose that we have a disk $E_{2}$ as in Figure 4.17. Then the arc $\varepsilon^{\prime}$ lies in some region of $S^{2}-N(R)$. Hence, considering the orientation of $R$, we see that one of the following occurs;

(1) The point (1) is bounded by the same Seifert circle as one of the points (3) and (5),

(2) The point (6) is bounded by the same Seifert circle as one of the points (2) and (4). In each case, we can find a $C$-product disk $E^{\prime}$ such that $\left|E \cap S^{2}\right|>\left|E^{\prime} \cap S^{2}\right|=0$ or 1 , a contradiction.

Figure 4.18 
Lemma 4.15. Let $E_{1}$ be as above. Then the following situation never occurs; The disk $E$ locally appears as in Figure 4.19, i.e., there is an outermost disk $F$ such that $\partial E-\left(E_{1} \cup F\right)$ has a component $\beta$ which contains no point of $\left(\partial E \cap S^{2}\right) \cup(\partial E \cap L)$.

\section{Figure 4.19}

Proof. Suppose there exists such a disk $F$. Let $\alpha$ be an $\operatorname{arc}$ in $E \cap S^{2}$ which cuts $F$ off $E$, and $\left(\mathfrak{a}_{s}, \mathfrak{a}_{t}\right)$ the label of $\alpha$ where $\mathfrak{a}_{s}$ is the label of an endpoint of $\beta$. First we examine the case where $E$ appears as in Figure 4.19 (a). If $s=i$ or 0 , we can find a $C$-product disk $E^{\prime}$ such that $\left|E^{\prime} \cap S^{2}\right|=1$, a contradiction (Figure 4.20 (a)). By Lemma 4.2, we have $s=0$ or $t=0$, and hence $t=0$. If $s=k$ or $l$, we can find a $C$-product disk $E^{\prime}$ such that $\left|E^{\prime} \cap S^{2}\right|=0$, a contradiction (see Figure 4.20 (b)). By Lemma 4.4, we have $s \neq m$. Then we see that $R$ locally appears as in Figure 4.20 (c). It is impossible that $\partial E$ runs toward the dealternator $\mathfrak{a}_{0}\left(=\mathfrak{a}_{t}\right)$ after passing through $\mathfrak{a}_{s}$ because of the orientation of $R$.

Second, we examine the case where $E$ locally appears as in Figure 4.19 (b). We can do this by the similar way to in the previous case. By Lemma $4.4, s \neq i$. If $s=0$ or $k$, we can find a $C$-product disk $E^{\prime}$ such that $\left|E^{\prime} \cap S^{2}\right|=0$, a contradiction. By Lemma 4.2 , we have $s=0$ or $t=0$, and hence $t=0$. If $s=l$ or $m$, we can find a $C$-product disk $E^{\prime}$ such that $\left|E^{\prime} \cap S^{2}\right|=1$, a contradiction. Then we see that it is impossible that $\partial E$ runs toward the dealternator $\mathfrak{a}_{0}\left(=\mathfrak{a}_{t}\right)$ before passing through $\mathfrak{a}_{s}$. See Figure 4.21. 4.15

Figure 4.20

Figure 4.21

Let $E_{1}^{\prime}=E-E_{1}$. Then $E_{1}^{\prime} \cap L$ is exactly one point, say, $x$. By Lemma $4.3, E_{1}^{\prime} \cap(E \cap$ $\left.S^{2}\right) \neq \emptyset$. By Lemmas 4.6 and 4.11, any arc of $E_{1}^{\prime} \cap\left(E \cap S^{2}\right)$ which does not separate $\varepsilon$ and $x$ is outermost in $E_{1}^{\prime}$. By Lemma 4.15, at least one of $E_{1}^{\prime} \cap\left(E \cap S^{2}\right)$ separates $\varepsilon$ and $x$. Among such separating arcs, let $\alpha$ be the one closest to $\varepsilon$. Then by Lemma 4.15 again, the subdisk of $E$ between $\varepsilon$ and $\alpha$ contains no arc of $E_{1}^{\prime} \cap\left(E \cap S^{2}\right)$. However, this contradicts Lemma 4.14. This completes the proof.

1.4. 


\section{Proof of Theorem 1.5}

In this section, we prove Theorem 1.5. Recall that a Seifert surface $R$ obtained by Seifert's algorithm is a union of Seifert disks and Seifert bands.

Definition. Let $R$ be a Seifert surface obtained by Seifert's algorithm. We say that two Seifert bands $B_{1}$ and $B_{2}$ of $R$ are parallel if they connect the same two Seifert disks.

The following is a case where we can deplumb a Hopf band from a fiber surface:

Proposition 5.1. Let $R$ be a fiber surface obtained by Seifert's algorithm. Suppose $R$ has a pair of parallel bands $B_{1}$ and $B_{2}$. Then, we can deplumb a Hopf band from $R$. Moreover, we have the following;

(1) the parallel bands are of the same sign, and

(2) for each $i=1,2$, we can cut the band $B_{i}$ by deplumbing a Hopf band from $R$, i.e., $R$ is a plumbing of $R-B_{i}$ and a Hopf band.

Proof. We denote by $L$ the link $\partial R$. We may assume that the Seifert circles, say, $C_{1}$ and $C_{2}$ connected by $B_{1}$ and $B_{2}$ bound mutually disjoint Seifert disks on the level 2-sphere $S^{2}$.

First, suppose the pair of parallel bands are of the same sign. We may assume they appear as in Figure 5.1 (a). We explicitly show that $R$ is a plumbing of a Hopf band and the surface $R-B_{i}$. Move $L$ by isotopy as in Figure 5.1 (a) and let $R^{\prime}$ be the surface as depicted. Apparently the Euler characteristic $\chi(R)$ is equal to $\chi\left(R^{\prime}\right)$. Hence by the uniqueness of fiber surfaces, we see that $R$ is isotopic to $R^{\prime}$. Now we can deplumb a Hopf band from $R^{\prime}$ as in Figure 5.1 (b). Then by retracing the above isotopy, we obtain the conclusion.

Next suppose that the pair of parallel bands are of the opposite signs, i.e., that the twisting of $B_{1}$ is opposite. Then by the isotopy as implied by Figure 5.1 (a), we can find a compressing disk for $R^{\prime}$, which contradicts the fact that fiber surfaces are of minimal genus and hence incompressible.

5.1

$$
\text { Figure 5.1 }
$$

The following proposition assures that if a diagram $D$ has a Seifert circle $C$ which contains an alternating tangle diagram, then any Seifert surface obtained by applying Seifert's algorithm to $D$ has parallel bands.

Proposition 5.2. Suppose a Seifert surface $R$ obtained from an alternating diagram $D$ is a fiber surface. Then $R$ has parallel bands. Moreover, if $D$ is reduced, then for any band $B$ of $R$, there is a band $B^{\prime}$ of $R$ which is parallel to $B$. 
Proof. By untwisting $R$ by isotopy if necessary, we may assume that $D$ is reduced. Moreover, we may assume that $D$ is unnested, because (1) by desumming along nested Seifert circles, we can decompose $R$ into fiber surfaces obtained from unnested alternating diagrams, and (2) if one of the decomposed surfaces has parallel bands, then so does $R$. Suppose a fiber surface $R$ for a link $L$ is obtained from a reduced unnested alternating diagram $D$. Then by [8] (or [2, Proposition 13.25]), $L$ is a connected sum of $(2, n)$-torus knots or links. Moreover the arguments in 8$]$ shows that $D$ is the 'standard' alternating diagram of a connected sum of $(2, n)$-torus knots or links. Hence we obtain the conclusion.

Proof of Theorem 1.5. The 'if' part follows from Theorem 1.1. We show the 'only if' part, using Corollary 1.6, by induction on the first Betti number $\beta_{1}$ of $R$. If $\beta_{1}(R)=1$, $R$ is a Hopf band, and hence the theorem holds. Assume the theorem holds for such surfaces with $\beta_{1}<k$, and let $R$ be a Seifert surface with $\beta_{1}(R)=k$ obtained from an almost alternating diagram $D$. By untwisting $R$ if necessary, we may assume that $D$ is reduced. By Corollary 1.6, we know how $R$ decomposes into Hopf bands. Hence by the following four lemmas, we will see that we can deplumb a Hopf band from $R$, in such a way that by deplumbing a Hopf band, we cut a band of $R$ corresponding to an alternator. Therefore the deplumbed surface satisfies the assumption of induction so that we see that $R$ is a Hopf plumbing.

1.5

Lemma 5.3. If $R$ desums along a nested Seifert circle, then we can cut a band of $R$ by deplumbing a Hopf band from $R$.

Proof. Suppose $D$ is nested, i.e., there exists a Seifert circle $C$ which contains another Seifert circle in both of its complementary regions in $S^{2}$. Then $R$ desums along $C$ into two surfaces, say, $R_{1}$ and $R_{2}$ such that $R_{1}$ is obtained from an alternating diagram and $R_{2}$ from an almost alternating diagram (cf. Proposition 2.5). Note that by Theorem 1.1, both $R_{1}$ and $R_{2}$ are fibers. By Proposition 5.2, we see that $R_{1}$ has parallel bands and hence so does $R$. Then by Proposition 5.1, we can cut a band of $R$ by deplumbing a Hopf band from $R$.

Lemma 5.4. If $R$ is a connected sum, then we can cut a band of $R$ by deplumbing a Hopf band from $R$.

Proof. Let $R$ be a connected sum of $R_{1}$ and $R_{2}$, where $R_{1}$ is obtained from an alternating diagram and $R_{2}$ from an almost alternating diagram by Proposition 2.4. Then by Theorem 1.1 and Proposition 5.2, $R_{1}$ has parallel bands, which are also parallel in $R$, 
and hence, by Proposition 5.1, we can cut a band of $R$ by deplumbing a Hopf band from $R$.

Lemma 5.5. If $R$ admits a decomposition of type (A), then we can cut a band of $R$ by deplumbing a Hopf band from $R$.

Proof. Suppose $R$ admits a decomposition of type (A). Then we can deform $R$ to $R^{\prime}$ by isotopy as depicted in Figure 5.2 (a), from which we can desum a fiber surface $R_{1}$ in Figure 5.2 (b). We can confirm that $R_{1}$ is obtained from an alternating diagram using Fact 2.3. By Proposition 5.2, $R_{1}$ has parallel bands. Though $R^{\prime}$ itself is not a surface obtained by Seifert's algorithm, we can apply the argument in the proof of Proposition 5.1, by regarding the inside of the dotted circle in Figure 5.2 (a) as a black box. Hence we can cut a band of $R^{\prime}$ (which is a band in the image of $R_{1}$ in $R^{\prime}$ ) by deplumbing a Hopf band from $R^{\prime}$. This corresponds to cutting a band of $R$ by deplumbing a Hopf band from $R$. Note that we can confirm that the surface obtained from $R$ by this cutting the band satisfies the assumption of induction.

\section{Figure 5.2}

Lemma 5.6. If $R$ admits a decomposition of type (B), then we can cut a band of $R$ by deplumbing a Hopf band from $R$.

Proof. According to whether the crossing visible in Figure 1.1 is an alternator or the dealternator, we have two cases. Let us call the former a decomposition of type (B1) and the latter of type (B2). Suppose that $R$ admits a decomposition of type (B1). Then by the same way as in the proof of Lemma 5.4, we can cut a band of $R$ by deplumbing a Hopf band from $R$.

Now assume $R$ does not admit a decomposition of type (B1). Then $R$ deplumbs into $R_{1}$ and $R_{2}$, which are both obtained from almost alternating diagrams (see Proposition 2.6). If $R_{1}$ or $R_{2}$ admits a decomposition of type (A), then we see, by the uniqueness of fiber surfaces, that $R$ also admits a decomposition of type (A), and the claim follows from Lemma 5.5. Hence we assume that neither $R_{1}$ nor $R_{2}$ admits a decomposition of type (A). Inductively, if we can do a decomposition of type (A) or (B1) in the process of desumming $R$ into a union of Hopf bands, then we see that $R$ also admits a decomposition of type (A) or (B1). So we assume that $R$ desums into a union of Hopf bands using decompositions of type (B2) alone. Then by another inductive argument, we see that $R$ is a pretzel surface of type $(1,-3, \ldots,-3)$ or $(-1,3, \ldots, 3)$. In this case, obviously we can cut a band of $R$ by deplumbing a Hopf band from $R$.

5.6 
Acknowledgment. The authors would like to thank Professor Taizo Kanenobu, Professor Tsuyoshi Kobayashi, Professor Yasutaka Nakanishi and Professor Makoto Sakuma for their comments. Part of this work was carried out while the first author was visiting at University of California, Davis. He would like to express thanks to Professor Abigail Thompson and the department for their hospitality.

\section{REFERENCES}

[1] C. Adams and et al: Almost alternating links, Topology and its Appl., 46 (1992), 151-165.

[2] G. Burde and H. Zieschang: Knots, Studies in Math. 5, Walter de Gruyter, 1985.

[3] R. Crowell, Genus of alternating link types, Ann. of Math., 69 (1959), 258-275.

[4] D. Gabai: Detecting fibred links in $S^{3}$, Comment. Math. Helv., 61 (1986), 519-555.

[5] J. Harer: How to construct all fibered knots and links, Topology, 21 (1982), 263-280.

[6] P. Melvin and H. Morton: Fibred knots of genus 2 formed by plumbing Hopf bands, J. London Math. Soc., (2) 34 (1986), 159-168.

[7] K. Murasugi, On the genus of the alternating knot, I, II, J. Math. Soc. of Japan, 10 (1958), 94-105, 235-248.

[8] K. Murasugi: On alternating knots, Osaka Math. J., 12 (1960), 277-303.

[9] K. Murasugi: On a certain subgroup of an alternating link, Amer. J. Math., 85 (1963), 544-550.

[10] D. Rolfsen: Knots and Links, Math. Lecture Series 7, Publish or Perish Inc., Berkeley, 1976.

[11] J. Stallings: On fibering certain 3-manifolds, In: Topology of 3-manifolds and related topics (Georgia, 1961) Prentice Hall (1962), 95-100.

Hiroshi Godaｇoda@math.kobe-u.ac.jp

Graduate School of Science and Technology, Kobe University,

Rokkodai 1-1, Nada, Kobe, 657-8501, Japan

Mikami Hirasawa hirasawa@math.sci.osaka-u.ac.jp

Department of Mathematics, Graduate School of Science,

Osaka University, Machikaneyama 1-1, Toyonaka, 560-0043, Japan

Ryosuke Yamamoto ryosuke@math.kobe-u.ac.jp

Graduate School of Science and Technology, Kobe University,

Rokkodai 1-1, Nada, Kobe, 657-8501, Japan 
This figure "Figure1-1.gif" is available in "gif" format from: http://arxiv.org/ps/math/9904043v1 
This figure "Figure2-1.gif" is available in "gif" format from: http://arxiv.org/ps/math/9904043v1 
This figure "Figure3-1.gif" is available in "gif" format from: http://arxiv.org/ps/math/9904043v1 
This figure "Figure4-1.gif" is available in "gif" format from: http://arxiv.org/ps/math/9904043v1 
This figure "Figure5-1.gif" is available in "gif" format from: http://arxiv.org/ps/math/9904043v1 
This figure "Figure2-2.gif" is available in "gif" format from: http://arxiv.org/ps/math/9904043v1 
This figure "Figure3-2.gif" is available in "gif" format from: http://arxiv.org/ps/math/9904043v1 
This figure "Figure4-2.gif" is available in "gif" format from: http://arxiv.org/ps/math/9904043v1 
This figure "Figure5-2.gif" is available in "gif" format from: http://arxiv.org/ps/math/9904043v1 
This figure "Figure2-3.gif" is available in "gif" format from: http://arxiv.org/ps/math/9904043v1 
This figure "Figure3-3.gif" is available in "gif" format from: http://arxiv.org/ps/math/9904043v1 
This figure "Figure4-3.gif" is available in "gif" format from: http://arxiv.org/ps/math/9904043v1 
This figure "Figure4-4.gif" is available in "gif" format from: http://arxiv.org/ps/math/9904043v1 
This figure "Figure4-5.gif" is available in "gif" format from: http://arxiv.org/ps/math/9904043v1 
This figure "Figure4-6.gif" is available in "gif" format from: http://arxiv.org/ps/math/9904043v1 
This figure "Figure4-7.gif" is available in "gif" format from: http://arxiv.org/ps/math/9904043v1 
This figure "Figure4-8.gif" is available in "gif" format from: http://arxiv.org/ps/math/9904043v1 
This figure "Figure4-9.gif" is available in "gif" format from: http://arxiv.org/ps/math/9904043v1 
This figure "Figure4-10.gif" is available in "gif" format from: http://arxiv.org/ps/math/9904043v1 
This figure "Figure4-11.gif" is available in "gif" format from: http://arxiv.org/ps/math/9904043v1 
This figure "Figure4-12.gif" is available in "gif" format from: http://arxiv.org/ps/math/9904043v1 
This figure "Figure4-13.gif" is available in "gif" format from: http://arxiv.org/ps/math/9904043v1 
This figure "Figure4-14.gif" is available in "gif" format from: http://arxiv.org/ps/math/9904043v1 
This figure "Figure4-15.gif" is available in "gif" format from: http://arxiv.org/ps/math/9904043v1 
This figure "Figure4-16.gif" is available in "gif" format from: http://arxiv.org/ps/math/9904043v1 
This figure "Figure4-17.gif" is available in "gif" format from: http://arxiv.org/ps/math/9904043v1 
This figure "Figure4-18.gif" is available in "gif" format from: http://arxiv.org/ps/math/9904043v1 
This figure "Figure4-19.gif" is available in "gif" format from: http://arxiv.org/ps/math/9904043v1 
This figure "Figure4-20.gif" is available in "gif" format from: http://arxiv.org/ps/math/9904043v1 
This figure "Figure4-21.gif" is available in "gif" format from: http://arxiv.org/ps/math/9904043v1 